\title{
Adición a la dieta de Metachirus nudicaudatus (Didelphimorphia: Didelphidae) en el Parque Nacional Altos de Campana, Panamá
}

\section{Ángel Sosa-Bartuano}

Sociedad Mastozoológica de Panamá, Ciudad de Panamá, Panamá

Colaborador Museo de Vertebrados de la Universidad de Panamá, Ciudad de Panamá, Panamá. asosa2983@gmail.com

La información sobre los hábitos alimenticios de la Zorra Morena (Metachirus nudicaudatus) es escasa (Lessa \& Geise 2010). Algunos trabajos sobre la dieta de M. nudicaudatus señalan que es una especie parcialmente frugívora (Hunsaker 1977, Lessa \& Costa 2010, Cáceres \& Lessa 2012, Lessa et al. 2013). Otros estudios indican que es altamente insectívora, consumiendo ocasionalmente vertebrados y frutos (Santori et al. 1995, De Carvalho et al. 1999, Vieira \& Astúa de Moraes 2003, Lessa \& Geise 2014). En trampas Tomahawk se han capturado individuos empleando como cebo bananas, aceite de hígado de bacalao, avena, mantequilla de maní y tocino (Cáceres 2004, Morães Junior 2004, Crouzeilles et al. 2010).

El 25 de julio de 2015 a las $21 \mathrm{~h} 20$ en el sendero La Rana Dorada del Parque Nacional Altos de Campana (PNAC), provincia de Panamá Oeste, Panamá ( $8^{\circ} 41^{\prime} 3 " \mathrm{~N}, 79^{\circ} 55^{\prime} 42^{\prime \prime W}$; 857 msnm; Figura 1) se observó un individuo de M. nudicaudatus alimentándose de frutos caídos de Otoba acuminata (Myristicaceae) (Figura 2). El animal cojeaba al desplazarse, lo que facilitó la aproximación para observarlo en detalle y fotografiarlo. Consumió durante siete minutos la pulpa de aproximadamente cinco frutos maduros ubicados en el suelo con la pulpa expuesta. La disponibilidad de frutos era alta, aproximadamente 25 en la base del árbol. El animal utilizó las patas delanteras para manipular y consumir los frutos sin levantarlos del suelo, dejándolos en el lugar donde los encontró. Cuando terminó se fue corriendo por el sotobosque aunque quedaban aún varios frutos sin consumir. Algunos trabajos sugieren que M. nudicaudatus tiene hábitos terrestres, prefiriendo forrajear y anidar en el suelo (Loretto et al. 2005), trepando solo en situaciones de peligro (Reid 2009). Este trabajo provee una observación adicional sobre sus hábitos terrestres.

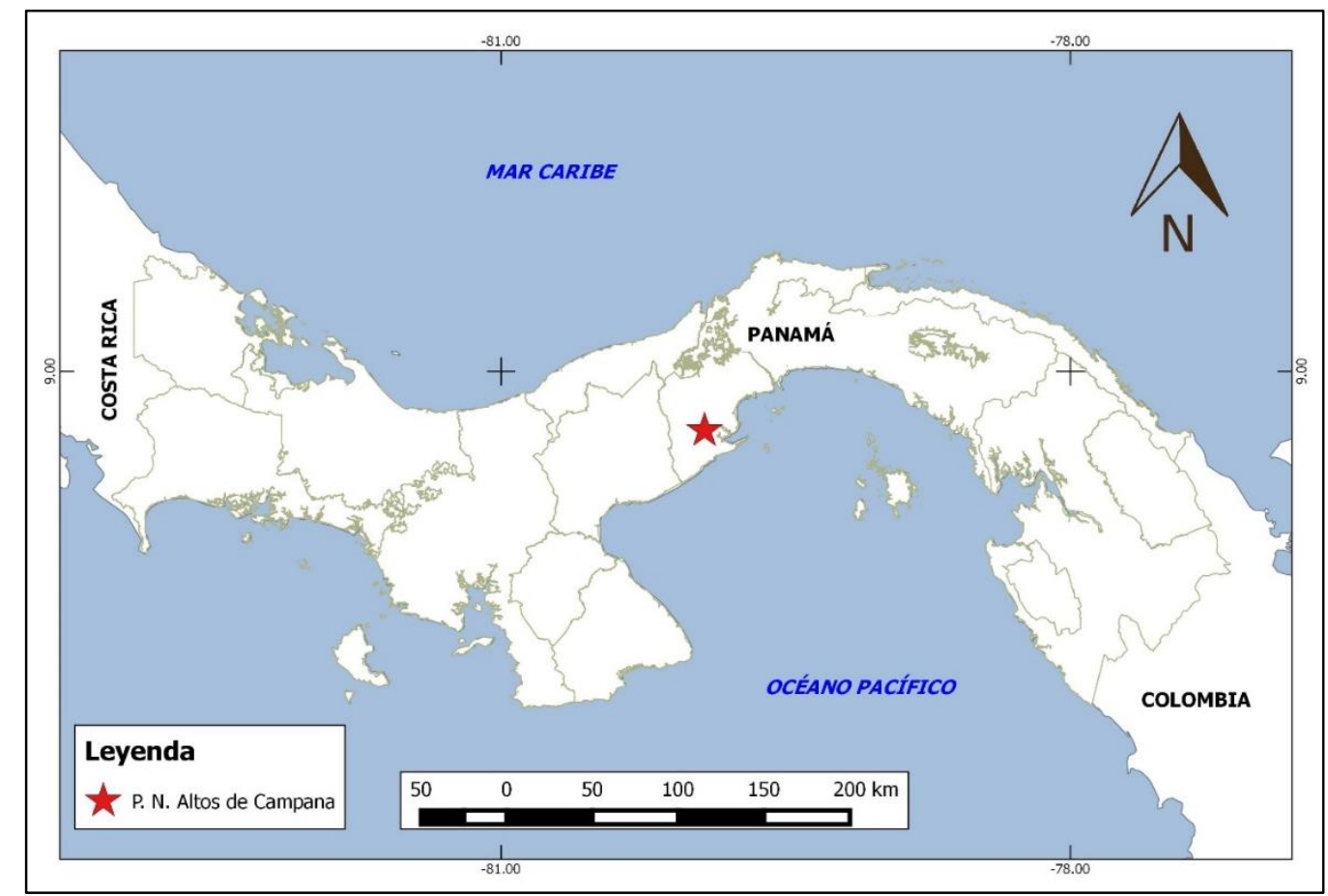

Figura 1. Sitio de observación del consumo de Otoba acuminata por la Zorra Morena Metachirus nudicaudatus (estrella roja) en el Parque Nacional Altos de Campana, provincia de Panamá Oeste, Panamá. 


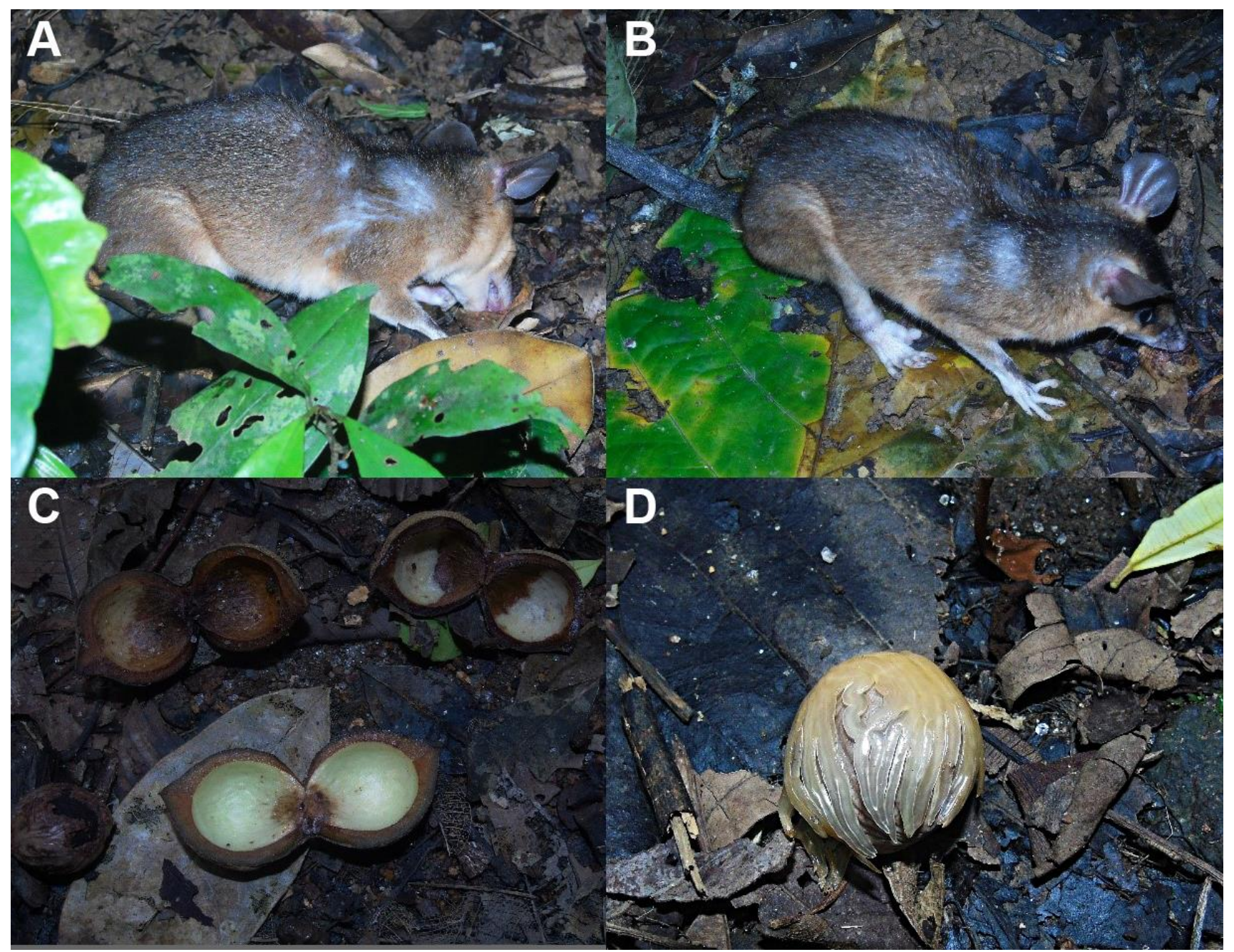

Figura 2. Zorra Morena (Metachirus nudicaudatus) consumiendo frutos de Otoba acuminata (A, B) en el sendero La Rana Dorada, Parque Nacional Altos de Campana, Panamá. Detalle de los frutos (C, D).

Se ha sugerido que los cambios estacionales marcados influyen en la composición de la dieta de M. nudicaudatus (Lessa \& Geise 2014). El hábito frugívoro de M. nudicaudatus se ha asociado con una alta disponibilidad de frutos durante los meses lluviosos (Lessa \& Geise 2014), patrón similar al observado en este trabajo ya que la observación se realizó en la estación lluviosa que en el PNAC tiene una duración promedio de nueve meses (IGNTG 1988).

En trabajos anteriores se han citado como parte de su dieta natural las siguientes familias de plantas: Araceae, Bromeliaceae, Cactaceae, Melastomataceae, Rubiaceae y Smilacaceae (Cáceres 2004, Lessa \& Geise 2014). Este es el primer registro de Myristicaceae en la dieta de M. nudicaudatus.

\section{Agradecimientos}

Agradezco a Eva Pinzón por el apoyo en campo, a Rodolfo Flores por la identificación de los frutos y a Rafael Samudio Jr. por proporcionar literatura.

\section{Mammalogy Notes | Notas Mastozoológicas \\ Sociedad Colombiana de Mastozoología \\ Vol. 3 Num. 1| 2016}


Referencias

CÁCERES, N. C. 2004. Diet of three didelphid marsupials (Mammalia: Didelphimorphia) in Southern Brazil. Mammalian Biology 69: 430-433.

CÁCERES, N. C. \& L. G. LESSA. 2012. O papel de marsupiais na dispersão de sementes. Pp. 407-426 in Os Marsupiais do Brasil: Biologia, ecologia e conservação (Campo Cáceres N.C., editor). Campo Grande, Editora UFMS, Brasil.

DE CARVALHO, F. M. V., et al. 1999. Diet of small mammals in Atlantic Forest fragments in southeastern Brazil. Revista Brasileira de Zoociências 1: 941-101.

CROUZEILLES, R., et al. 2010. Philander frenatus e Metachirus nudicaudatus: Competição ou necessidades ecológicas diferentes na Floresta Atlântica. Mastozoologia Neotropical 17: 135-140.

EMMONS, L. \& F. FEER. 1990. Neotropical Rainforest Mammals. A Field Guide. The University of Chicago Press. Chicago. 307 pp.

FREITAS, S. R., et al. 1997. Habitat preference and food use by Metachirus nudicaudatus and Didelphis aurita (Didelphimorphia, Didelphidae) in a restinga forest at Rio de Janeiro. Revista Brasileira de Biologia 57:93-98.

HANDLEY, C.O. Jr. 1976. Mammals of the Smithsonian Venezuelan Project. Brigham Young University Science Bulletin, Biological Series 20 : 1-89.

HUME, I. 2006. Nutrition and Digestion. Pp. 137-138 in Marsupials. (Armati P. et al. editors). Cambridge University Press, University of Sidney, Australia.

HUNSAKER, D., II. 1977. Ecology of New World marsupials. Pp. 95-156 in The Biology of Marsupials (Hunsaker II, D. editor). Academic Press, New York, USA.

IGNTG (Instituto Geográfico Nacional Tommy Guardia). 1988. Atlas Nacional de la República de Panamá. Tercera edición. 222 pp.

LESSA, L. G. \& F. N. COSTA. 2010. Diet and seed dispersal by five marsupials (Didelphimorphia: Didelphidae) in a Brazilian cerrado reserve. Mammalian Biology 75: 10-16.

LESSA, L. G. \& L. GEISE. 2010. Hábitos alimentares de marsupiais didelfídeos brasileiros: análise do estado de conhecimento atual. Oecologia Australis 14: 918-927.

LESSA, L. G. \& L. GEISE. 2014. Food habits of Metachirus nudicaudatus (Didelphimorphia, Didelphidae) in a Brazilian Cerrado: diet composition and dietary seasonality. Studies on Neotropical Fauna and Environment 49: 75-78.

LESSA L. G., et al. 2013. Effects of gut passage on the germination of seeds ingested by didelphid marsupials in a Neotropical savanna. Acta Botanica Brasilica 27: 519-525. LORETTO, D., et al. 2005. Defense behavior and nest architecture of Metachirus nudicaudatus Desmarest, 1817 (Marsupialia, Didelphidae). Mammalia 69: $417-419$.

MEDELLÍN, R. A., et al. 1992. Noteworthy record of three mammals from Mexico. The Southwestern Naturalist 37: 427-429.

MORÃES JUNIOR, E. A. 2004. Radio Tracking of one Metachirus nudicaudatus (Desmarest 1817) Individual in Atlantic Forest of Southeastern Brazil. Boletim Museu Biologia Mello Leitão 17: 57-64.

NAVARRO, J. C. 2001. Parques Nacionales - Panamá. Segunda Edición. Ediciones Balboa S. A., Panamá. 221 pp.

REDFORD, K. H. \& J. F. EISENBERG. 1992. Mammals of the Neotropics, Volume II. University of Chicago Press, Chicago, USA. 435 pp.

REID, F. 2009. A Field Guide to the Mammals of Central America and Southeast Mexico. Second Edition. Oxford University Press, USA. 346 pp.

SANTORI, R. T. et al., 1995. Diet composition of Metachirus nudicaudatus and Didelphis aurita (Marsupialia, Didelphoidea) in southeastern Brazil. Mammalia 59: 511-516.

VIEIRA, E. M. \& D. ASTÚA DE MORAES. 2003. Carnivory and insectivory in Neotropical marsupials. Pp. 271-284 in Predators With Pouches: The Biology of Carnivorous Marsupials. (Jones, M. et al. editors). CSIRO Publishing, Collingwood, Australia. 